\title{
METEOROID ORBITS AVAILABLE FROM THE \\ IAU METEOR DATA CENTER
}

\author{
B.A. LINDBLAD \\ Lunds Observatorium, Box 43, S-22100 Lund, Sweden \\ E-mail : linasu@gemini.ldc.lu.se
}

D.I. STEEL

Anglo-Australian Observatory, Private Bag, Coonabarabran, NSW 2357; and
Department of Physics and Mathematical Physics, University of Adelaide,
G.P.O. Box 498, Adelaide, SA 5001, Australia
E-mail : DIS@AAOCBN3.AAO.GOV.AU

Abstract. Since it was founded early in the 1980's, the IAU Meteor Data Center (IAU MDC) has accumulated a large number of the meteoroid orbits measured worldwide so as to make these freely available to all interested researchers. The total number of orbits available is about 68,000 , of which about 6,000 were determined using optical techniques (photographic or TV), the bulk having been detected using decameter radars. The observation sites range from various locations in the U.S.A., Canada, Australia, and in the former Soviet Union and Czechoslovakia; radar orbits from the Soviet Equatorial Expedition to Somalia are also archived. About 39,000 of the 62,000 radar orbits are derived from the Harvard Radar Meteor Project. Most of these programs were carried out during the 1960's and 1970's, but still represent our best knowledge of the orbital distribution of interplanetary particles in the size range from $100 \mu \mathrm{m}$ to 1 meter. A new survey currently in progress in New Zealand has so far rendered over 350,000 orbits, and it is anticipated that these will soon become available through the IAU MDC. Presently the 68,000 orbits archived in the IAU MDC are only available on magnetic recording media, but it is planned that they will shortly be made accessible via anonymous $\mathrm{ftp}$.

\section{The IAU Meteor Data Center}

Starting in the 1930's it became possible to determine the orbits of individual particles observed as meteors using photographic techniques, so that at least the nighttime meteor showers could be investigated and definitively linked to specific comets. The development of suitable (decameter) radars in the 1940's meant that by the 1950's the daytime showers were also amenable to study, and the smaller particles (fainter meteors) detectable with such radars also led to a much enhanced detection rate. During the 1950's the various researchers at the Jodrell Bank station of the University of Manchester (England) developed a set-up which could render meteoroid orbits by determining the atmospheric speed from Fresnel oscillations in the echo amplitude, and using the time of closest approach to each of a series of receiver stations separated by several kilometers to deduce the meteor radiant. With the time of detection known the heliocentric orbit could then be found. The Jodrell Bank team determined over 2,500 orlsits in this way, comprising the largest repository of small particle orbits available in the late 1950 's for investigations of the 
origin and evolution of such bodies. Some hundreds of orbits of larger meteoroids had also been measured using photographic methods, in particular by the team at Harvard led by F.L.Whipple.

These pioneering Jodrell Bank orbits have unfortunately been lost over the years, and so are no longer available to researchers wanting to investigate their orbital distribution, nor indeed historians of astronomy. With the realization that such epoch-making data are of importance, and should not be lost since they are still of scientific value, the first author of this paper established the IAU Meteor Data Center with a view to ensuring that all subsequent meteoroid orbit data be archived for use by new researchers. Five immediate justifications for such a data center spring to mind : (i) Such meteor research programs are ephemeral, so that unless a central respository exists, as a program terminates and the researchers disperse the data are often mislaid or deliberately discarded; (ii) Although several programs were carried out during the years $1960-75$, since that time there has been very little activity in this area, making the old data the only available data; (iii) Some millions of dollars and roubles were spent in obtaining the data initially, so that it would not be sensible to let them be lost for the sake of a few thousand units of the same currencies, which is all that was required to assure their security; (iv) New techniques (and new minds) have made alternative types of analysis possible, in particular with the huge improvement in the computing power which has become available; (v) Newly-discovered comets and asteroids present themselves as possible parent objects for previously-observed meteoroids, the original orbits being needed for testing of such genetic relationships.

In fact the decision to collect all meteoroid orbits together was made only just in time. For example, well over $50 \%$ of the orbits now archived come from the Harvard Radar Meteor Project observation campaigns of 1961-65 and 1968-69, and the only source for these available was two computer tapes which had been stored in Z. Sekanina's attic in Pasadena, California, for some years. Thankfully those tapes were successfully read by C.D. Murray and S.F. Dermott, and the data saved. Similarly the orbits from the Adelaide radar surveys of 1960-61 and 196869 , the only such data collected from the southern hemisphere until very recently, were rescued from racks of dusty and decaying cards in an old data store which was cleared out soon thereafter.

Despite some close escapes most of the known orbits have been collected together, with Lindblad basing the IAU MDC at the Lund Observatory in Sweden (Lindblad, 1991a). Its foundation results from a proposal to the IAU at the General Assembly of 1976 from Commission 22, confirmed at the GA in 1982.

\section{Meteoroid orbits available}

The meteoroid orbits available from the IAU MDC in machine-readable form are listed in Table 1. These have been divided here into two separate groups, according to whether the meteors were observed optically (i.e. nighttime meteors only) or with radars (both night- and day-time observations). The survey names are given here only for the purposes of easy identification. A complete description of the observing techniques, sites, references to original publications on each, etc., is available from the IAU MDC (see below). 
Table 1 : Meteoroid orbits archived at the

IAU Meteor Data Center.

\begin{tabular}{|c|c|c|}
\hline Survey & Operation & Orbits \\
\hline \multicolumn{3}{|c|}{ Optical Surveys } \\
\hline Harvard P Photo ${ }^{1}$ & $1936-59$ & 1,245 \\
\hline Harvard G Photo ${ }^{2}$ & $1952-54$ & 2,529 \\
\hline Prairie Network $^{3}$ & $1963-75$ & 336 \\
\hline MORP & $1971-84$ & 218 \\
\hline Ondřejov 5 & $1947-89$ & 285 \\
\hline Soriet Photo ${ }^{6}$ & $1940-83$ & 1,111 \\
\hline TV meteors ${ }^{7}$ & $1982-84$ & 531 \\
\hline Japanese small camera $^{8}$ & $1964-89$ & 325 \\
\hline \multicolumn{3}{|c|}{ Radar Surveys } \\
\hline Adelaide $^{9}$ & $1960-61$ & 2,092 \\
\hline Harvard ${ }^{10}$ & $1961-65$ & 19,327 \\
\hline Obninsk ${ }^{11}$ & $1967-68$ & 9,358 \\
\hline Adelaide $^{12}$ & $1968-69$ & 1,667 \\
\hline Harvard $^{13}$ & $1968-69$ & 19,818 \\
\hline Mogadisho $^{14}$ & $1968-70$ & 5,328 \\
\hline Kharkov $^{15}$ & 1975 & 5,317 \\
\hline
\end{tabular}

${ }^{1}$ Harvard Precisely-reduced photographic meteors, observed from Massachusetts and New Mexico.

${ }^{2}$ As above, except for with a less-accurate Graphical reduction. Some of the meteors in this catalogue are in fact also included in the precise orbits.

${ }^{3}$ Prairie Network fireballs, U.S.A.

4 Meteorite Orbit and Recovery Program fireballs, Canada.

5 Fireballs observed from Ondřejov Observatory in the Czech Republic (formerly part of Czechoslovakia), one station of the present European Network of fireball observatories. Other fireball orbits are also available directly from Ondřejov; see text.

- These precisely-reduced photographic orbits were observed from Dushanbe, Odessa and Kiev, in the former Soviet Union.

7 Television meteors (generally fainter than observed in the photographic surveys) recorded from Ontario, Canada.

8 Japanese $35 \mathrm{~mm}$ camera orbits (Koseki et al., 1990; Lindblad, 1991b).

- Radar meteors observed near Adelaide, Australia.

${ }^{10}$ Harvard Radar Meteor Project (RMP); observatory site near Havana, Illinois. Very faint radar meteors.

11 Obninsk, south of Moscow, Russia.

12 Second Adelaide survey, fainter limiting magnitude.

13 Harvard RMP Synoptic Year study.

14 Observatory built near Mogadisho, Somalia, by Soviet Equatorial Expedition; all catalogued orbits have southern radiants.

${ }^{15}$ Kharkov radar survey of faint meteors. Another 150,000 orbits have yet to be supplied to the IAU MDC. 
The optical meteors cover a wide range of initial particle sizes, from fireballs having masses of a kilogram or more down to faint TV-detected meteors having masses of order $0.1 \mathrm{mg}$ (i.e. sizes from a decimeter down to a millimeter). A description of all orbits determined photographically has been given by Lindblad (1991a).

Turning to the radar orbits, the masses of the larger particles detected (corresponding to limiting radar magnitudes of $+6 \div 8$, as for the Adelaide, Obninsk and Mogadisho surveys) are similar to those of the TV meteors. However, the Kharkov and Harvard surveys detected meteors as faint as magnitude $+12 \div 13$ (corresponding to meteoroids of masses down to $\sim 1 \mu \mathrm{g}$, or sizes of $\sim 100 \mu \mathrm{m}$ ).

It has generally been found in orbit programs that distinct streams/showers provide $30 \%$ or more of the total number of orbits, with a reducing fraction in streams as fainter meteors (smaller meteoroids) are accessed in surveys using more powerful radars. The personal experience of the second author in manipulating these data has confirmed this to be the case; he finds that streams/showers are more preponderant amongst the Adelaide and Obninsk orbits, compared to the other radar surveys. For typical orbital distribution plots, see Steel (1991).

\section{Other meteoroid orbits}

Apart from the surveys listed in Table 1, there are some other photographic orbits available from the IAU MDC, although not all have yet been added to the checked and formatted repository. These include 25 orbits from the program at New Mexico State University in the 1970's. The 325 orbits determined by Japanese amateur groups using $35 \mathrm{~mm}$ cameras (Koseki et al., 1990; Lindblad, 1991a,b) are a recent addition. In fact there are a number of amateur groups elsewhere who are now producing excellent sets of orbits, usually for specific streams such as the Perseids and the Geminids; for references see Lindblad (1991a).

There are also some hundreds of fireball orbits measured from the Ondrejov Observatory (251 65 Ondřejov, Czech Republic) which are available directly from that institution; the appropriate contact person is P.Spurný.

After development stretching through the 1980's, a new powerful meteoroid orbit radar began operations in New Zealand in 1990. This has so far rendered over 350,000 individual orbits to radar magnitude $+12 \div 13$ (Baggaley et al., 1993, 1994). Although observing campaigns to investigate background/sporadic counts have been run, much of the data has been collected during the epochs of specific showers; in particular the $\eta$ Aquarids and tho $\delta$ Aquarids. The present antenna pattern results in most detected meteors having radiants between declinations of $+5^{\circ}$ and $-40^{\circ}$, but this will soon be reversed so as to give coverage to the south equatorial pole, since deep southern radiants are very poorly represented in the data presently available. It is anticipated that these orbits will be made available through the IAU MDC in the future. 


\section{How to obtain the data}

The meteor orbits listed in Table 1 are available directly from B.A.Lindblad at the address given at the head of this paper, in the form of a nine-track 1600 bpi tape. Email contact may be made through linasu@gemini.ldc.lu.se. Geophysical data are also available for most of the photographic orbits (e.g. start and end heights of the meteor trains, magnitudes, etc.).

If some other data format (e.g. exabyte, 3.5- or 5.25-inch disks) is required, the interested party should approach D.I.Steel; email contact is preferable, through either diseaaocbn3.aao.gov.au or dsteelephysics.adelaide.edu.au.

It is planned that these data will be available through anonymous $\mathrm{ftp}$ at some stage during 1994. Even if this is the case, it will likely be highly desirable to any researcher to have access to the paper documentation on the data sets, and this documentation is obtainable from B.A.Lindblad.

\section{Acknowledgements}

Various scientists have kindly made these data available to the general astronomical community, either through allowing their release or else searching out the data from where they were stored and supplying them to the IAU MDC in a machinereadable format. Financial support for the center has come from the International Astronomical Union, the Swedish Natural Science Research Council, and the Kungliga Fysiografiska Sällskapet. D.I.S. checked and formatted the radar orbits at the IAU MDC in 1987 when he was supported by the European Space Agency; his subsequent work on the data has been supported by the Australian Research Council.

\section{References}

Baggaley, W.J., R.G.T.Bennett, D.I.Steel \& A.D.Taylor (1994), "The Advanced Meteor Orbit Radar Facility : AMOR." Ql. J. Roy. Astron. Soc., 35, in press.

Baggaley, W.J., Taylor, A.D. and Steel, D.I. : 1992, "The Southern Hemisphere Meteor Orbit Radar Facility : AMOR." In Meteoroids and their parent bodies (J. Štohl and I.P. Williams, Eds.), 245-248, Astron. Inst., Slovak Acad. Sci., Bratislava, Slovakia.

Koseki, M., Sekiguchi, T. and Ohtsuka, K. : 1990, "Photographic meteor observations in Japan.". In Asteroids, Comets, Meteors III (C.-I. Lagerkvist, H. Rickman, B.A. Lindblad and M. Lindgren, Eds.), 547-550, University of Uppsala, Sweden.

Lindblad, B.A. : 1991a, "The IAU Meteor Data Center in Lund." In IAU Colloq. 126 : Origin and Evolution of Interplanetary Dust (A.C. Levasseur-Regourd and H. Hasegawa, Eds.), 311-314, Kluwer, Dordrecht, Holland; reprinted in IAU Information Bulletin, 70, 10-13 (1993).

Lindblad, B.A. : 1991b, "A study of meteor orbits obtained in Japan." In IAU Colloq. 126 : Origin and Evolution of Interplanetary Dust (A.C. Levasseur-Regourd and H. Hasegawa, Eds.), 299-302, Kluwer, Dordrecht, Holland.

Steel, D. : 1991, "The orbital distribution and origin of meteoroids." In IAU Colloq. 126 : Origin and Evolution of Interplanetary Dust (A.C. Levasseur-Regourd and H. Hasegawa, Eds.), 291-298, Kluwer, Dordrecht, Holland. 